\title{
Burnout e saúde mental em tempos de pandemia de COVID -19: revisão sistemática com metanálise
}

RESUMO | OBJETIVO: Identificar os impactos gerados pela pandemia na saúde mental dos profissionais enfermeiros. MÉTODO: Revisão sistemática com metassíntese, onde foi realizada uma busca nas bases de dados Google acadêmico e a Biblioteca Virtual em Saúde (BVS/SCIELO). Foram utilizados os Descritores em Ciências da Saúde Burnout, esgotamento profissional, pandemia e COVID-19, fazendo uso do operador booleano AND. RESULTADOS: Após a realização da busca nas bases de dados mencionadas com os descritores elencados, foram encontrados 29 estudos. A posteriori foram aplicados os critérios de inclusão e exclusão resultando em uma amostra final de 10 estudos. CONCLUSÃO: Cargas horárias exaustivas, más condições de trabalho, deficiência no dimensionamento de pessoal, poucos recursos materiais, desvalorização e baixa remuneração. Esses fatores, associados ao medo de transmitir a doença para familiares e amigos, o isolamento social e outros aumentam o risco de desenvolvimento de problemas psicológicos causando efeitos deletérios à saúde mental.

Palavras-chaves: Enfermagem; Saúde Mental; Pandemia; COVID-19.

\begin{abstract}
OBJECTIVE: To identify the impacts generated by the pandemic on the mental health of professional nurses. METHOD: Systematic review with meta-synthesis, where a search was performed in the Google academic databases and the Virtual Health Library (VHL/SCIELO). Descriptors in Health Sciences Burnout, professional exhaustion, pandemic and COVID-19 were used, making use of the Boolean operator AND. RESULTS: After conducting the search in the databases mentioned with the listed descriptors, 29 studies were found. A posteriori, the inclusion and exclusion criteria were applied, resulting in a final sample of 10 studies. CONCLUSION: Exhaustive workloads, poor working conditions, deficiency in staffing, few material resources, devaluation and low remuneration. These factors, associated with the fear of transmitting the disease to family and friends, social isolation and others increase the risk of developing psychological problems causing deleterious effects on mental health.

Keywords: Nursing; Mental health; Pandemic; COVID-19.

RESUMEN | OBJETIVO: Identificar los impactos generados por la pandemia en la salud mental de los profesionales de enfermería. MÉTODO: Revisión sistemática con metástasis, donde se realizó una búsqueda en las bases de datos académicas de Google y en la Biblioteca Virtual de Salud (VHL/SCIELO). Se utilizaron los Descriptores en Ciencias de la Salud Burnout, Professional Exhaustion, Pandemic y COVID-19, utilizando el operador booleano AND.RESULTADOS: Después de buscar en las bases de datos mencionadas con los descriptores enumerados, se encontraron 29 estudios. Se aplicaron criterios a posteriori, inclusión y exclusión, lo que dio lugar a una muestra final de 10 estudios. CONCLUSIÓN: Cargas de trabajo exhaustivas, malas condiciones de trabajo, deficiencia en la dimensionamiento del personal, pocos recursos materiales, devaluación y baja remuneración. Estos factores, asociados con el miedo a transmitir la enfermedad a familiares y amigos, el aislamiento social y otros aumentan el riesgo de desarrollar problemas psicológicos que causan efectos perjudiciales en la salud mental.
\end{abstract}

Palabras claves: Enfermería; Salud Mental; Pandemia; Covid-19.

\section{Dayse Christina Rodrigues Pereira Luz}

Pós Doutoranda em Ciências da Saúde pelo Centro Universitário Saúde ABC - FMABC. Docente da Faculdade de Juazeiro do Norte - FJN. ORCID: 0000-0002-5719-3574

\section{José Rafael Eduardo Campos}

Graduado em Enfermagem pelo Centro Universitário de Juazeiro do Norte - UNIJUAZEIRO. Membro do Grupo de Pesquisa em Ciências da Saúde do CNPq e Residente Multiprofissional com Ênfase em Neurologia e Neurocirurgia pela Escola de Saúde Pública HRC - ESP.

ORCID: 0000-0003-0463-4839

\section{Pabllo de Oliveira Saraiva Bezerra}

Graduando em Medicina pela Faculdade de Medicina de Juazeiro do Norte - Estácio e Membro Diretor da Liga Acadêmica de Neurocirurgia do Cariri - LANCIR.

ORCID: 0000-0003-0275-1329

\section{Jessika Brenda Rafael Campos}

Graduada em Enfermagem pelo Centro Universitário de Juazeiro do Norte - UNIJUAZEIRO e Residente Multiprofissional com Ênfase em Transplante pelo Hospital Universitário Walter Cantídio HUWC - UFC. ORCID: 0000-0003-3768-5480

\section{Anderson Marcos Vieira do Nascimento \\ Mestre em Saúde Pública pela Universida- de Estadual da Paraíba UEPB. \\ ORCID: 0000-0001-8575-1580}

Recebido em: 11/03/2021

Aprovado em: 05/04/2021

\section{Alyce Brito Barros}

Graduanda em Enfermagem pelo Centro Universitário de Juazeiro do Norte - UNIJUAZEIRO.

ORCID: 0000-0002-2775-1264

INTRODUÇÃO

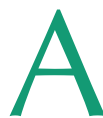
Organização Mundial da Saúde (OMS), em dezembro de 2019, notificou a identificação de um novo vírus detectado pelas autoridades chinesas em um paciente hospitalizado com pneumonia na cidade de Wuhan, posteriormente esse vírus foi denominado de SARS-CoV-2, causador da doença COVID-19. Após 5 meses o 
vírus contaminou milhões de pessoas $e$ levou milhares a óbito promovendo uma pandemia sem precedentes na atualidade. A doença se manifesta através de uma infecção viral aguda com alta taxa de transmissibilidade que acontece principalmente pelas vias respiratórias através da inalação de gotículas e/ou aerossóis e, também, através do contato direto entre pessoas e objetos que estejam contaminados1,2.

Responsável por causar diversos sintomas, entre eles, sintomas respiratórios e digestivos, a COVID-19 apresenta uma manifestação que pode alternar desde uma patologia autolimitada leve até uma pneumonia grave, insuficiência respiratória aguda e choque séptico. Nesse sentindo, a falta de um tratamento especifico e a ausência de uma vacina somado ao aumento exponencial de casos, vem causando um elevado número de internações e óbitos, resultando em um aumento da procura dos sistemas de saúde levando a uma sobrecarga de trabalho dos recursos humanos3.

Dados emitidos pelos primeiros países afetados pela pandemia relatam que $40 \%$ das pessoas infectadas sofrerão uma doença leve, 40\% experimentarão doenças moderadas, como pneumonia, $15 \%$ sofrerão doença grave e $5 \%$ sofrerão doença grave, podendo resultar em óbito. A pandemia atinge os serviços de saúde exigindo uma demanda extra de estrutura, insumos e profissionais. Historicamente o financiamento da saúde no Brasil é insuficiente, com a instalação da pandemia no território brasileiro os serviços de saúde passaram a enfrentar sérias adversidades somando um total de 5.781.582 casos confirmados e 164.281 óbitos confirmados pela COVID-194,1,5.

Inúmeros fatores modificaram o ambiente laboral dos profissionais de saúde, sendo eles a falta de Equipamentos de Proteção Individual (EPI); o número restrito de leitos e ventiladores mecânicos; falta de conhecimento sobre a patologia e de treinamento para atender o paciente confirmado ou com suspeita de
COVID-19; ausência de um tratamento eficaz para a doença e alto nível de complexidade e gravidade dos pacientes; e necessidade em lidar e enfrentar um volume de óbitos elevado. Nesse sentindo, os profissionais de saúde formam um grupo de risco para a infecção, uma vez que estão expostos a fatores que promovem uma maior vulnerabilidade para adquirir a doença. Os riscos englobam uma maior exposição a patógenos, longas jornadas de trabalho, sofrimento psicológico, síndrome do esgotamento físico e mental inerente ao trabalho (síndrome de Burnout), fadiga e estigma6,7.

Aproximadamente 18.870 profissionais de enfermagem vivenciam a doença ou a suspeita dela, sendo que, 341 estão internados e 18.341 em quarentena. O número de óbitos é de 194 no território brasileiro8.

Mesmo sendo uma patologia recente, já se consegue observar os efeitos negativos da COVID-19 para a saúde mental dos profissionais de saúde. A enfermagem apresenta uma maior suscetibilidade ao desenvolvimento de problemas de saúde mental, esse fato se justifica devido ao maior tempo de convivência e interação com os pacientes; pressão para realizar suas atividades com qualidade; e o enfrentamento do processo de morte e morrer, uma vez que são profissionais treinados e capacitados para curar. Somado a isso, a alta carga de trabalho, sensação de apoio inadequada, alta taxa de infecção pelo COVID-19 entre os trabalhadores, medo de contaminar familiares e casos de óbitos na própria equipe de saúde são os principais fatores para resultados psicológicos adversos3,9.

No contexto da pandemia do COVID-19, a OMS observou que os profissionais de enfermagem pressionados com a situação atual apresentaram elevados níveis de ansiedade, somado ao risco de adoecer, causando drásticos problemas de saúde mental e aumentando os casos de Síndrome de Burnout (SB), além de também promover ansiedade, depressão e estresse4.
Dito isso, a seguinte pergunta norteadora tornou-se importante dentro do atual contexto vivenciado pelos profissionais de enfermagem: Quais os impactos da pandemia de COVID-19 na saúde mental dos enfermeiros?

Nesse sentindo, este estudo objetiva identificar os impactos gerados pela pandemia na saúde mental dos profissionais enfermeiros.

\section{MÉTODO}

Trata-se de uma revisão sistemática com metassíntese, onde foi realizada uma busca nas bases de dados Google acadêmico e a Biblioteca Virtual em Saúde (BVS/SCIELO). Foram utilizados os Descritores em Ciências da Saúde Burnout, esgotamento profissional, pandemia e COVID-19, fazendo uso do operador booleano AND.

Foram elencados os estudos de revisões, originais, carta ao editor que estavam disponíveis na integra de forma gratuita, publicados entre 2019 e 2020, nos idiomas inglês e português, e que abordassem a saúde mental dos profissionais de saúde durante o período da pandemia. Foram excluídos os artigos duplicados, projetos, resumos, carta ao editor e aqueles que não se enquadravam na temática abordada, para tal, foi realizada uma leitura minuciosa dos títulos e resumos, afim de excluir esses estudos.

$\mathrm{O}$ instrumento utilizado como referência para demonstrar o processo de busca e seleção dos estudos foi o Preferred Reporting Items for Systematic Review and Meta-Analyses (http://www. prisma-statement.org/)10.

Os riscos de viés dos estudos elencados foram analisados segundo a escala Downs and Black (1998)11 que é estruturada pela análise da qualidade do estudo (10 itens); capacidade de generalizar resultados do estudo (3 itens); viés do estudo (7 itens); determinação de viés pela amostra (6 itens) e determinação de achados ao acaso (1 item).

A partir das informações coletadas 
foi estruturada uma tabela com os dados identificados referentes ao autor/ano do estudo, objetivo, principais achados e a pontuação na escala Downs and Black (1998)11.
RESULTADOS

Após a realização da busca nas bases de dados mencionadas com os descritores elencados, foram encontrados 29

\section{FIGURA 1: Fluxograma de seleção dos estudos a partir do protocolo PRISMA}

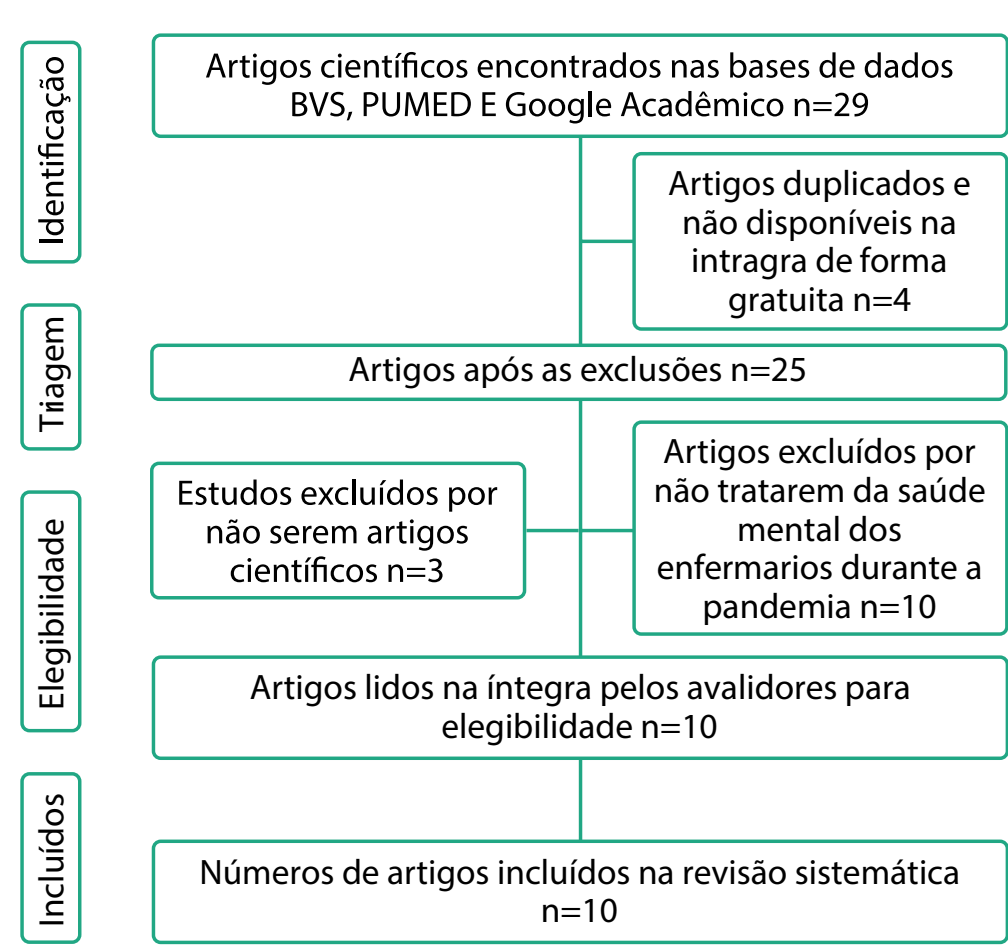

FONTE: Elaborado pelos autores

\section{TABELA 1: Risco de viés dos estudos selecionados}

AUTOR/ANO

OBJETIVO

Analisar a produção científica sobre a saúde dos trabalhadores da saúde que atendem pacientes no contexto da pandemia de COVID-19.

\section{PRINCIPAIS ACHADOS}

PONTUACCÃO NA ESCALA DOWNS AND BLACK

0 conhecimento dos enfermeiros e médicos sobre a doença foi considerado adequado; ausência de treinamento para assistência em saúde mental para pacientes com COVID-19 e que o sentimento de medo é uma realidade entres os profissionais que compõem essa equipe de saúde; profissionais de saúde representam 3,8\% a 20\% da população infectada no Brasil; profissionais da linha de frente experimentaram maior impacto em sua saúde mental, demonstrando como um fator de risco para os sintomas de ansiedade, depressão, insônia, angustia e medo; profissional de enfermagem do sexo feminino esteve associado a sintomas graves de depressão, angústia e ansiedade. 
Descrever e discutir a atuação dos profissionais

MOREIRA, de enfermagem, sua exposição aos fatores de

2020 risco no trabalho e a importância do apoio psicossocial na pandemia da COVID-19.
A enfermagem enfrenta problemas associados com a sobrecarga de trabalho, más condições de trabalho, falta de recursos e baixa valorização profissional; o número elevado de profissionais contaminados e afastados contribui para a sobrecarga das equipes de saúde e contribui para

o esgotamento psíquico da equipe de enfermagem; 0 impacto psicossocial desses profissionais também é elevado, principalmente, no sexo feminino; 0 estresse ocupacional é um importante indicador de exaustão psíquica refletindo na saúde mental dos profissionais de enfermagem; existem relatos adoecimento psíquico como ansiedade generalizada, depressão, ataques de pânico, síndrome de Burnout ou esgotamento profissional.

A jornada de trabalho é um fator que gera estresse entre os profissionais da saúde, sendo a sobrecarga de trabalho, em um momento de pandemia, um fator que pode levar ao esgotamento profissional; medidas como diálogo, comunicação entre profissionais e chefes, melhoria da alimentação, propagação de histórias exitosas, gestão e educação são estratégias elencadas para diminuir os impactos psíquicos.

Os enfermeiros enfrentam situações de violência física, verbal e psicológica e, apenas $29 \%$ desses profissionais se sentem seguros em seus ambientes de trabalho; os enfermeiros durante a pandemia enfrentam os fatores do alto risco de contaminação, do adoecimento, da morte, da possibilidade de infectar outros indivíduos, angústia, esgotamento, exposição a mortes em ampliadas proporções, decepção de não conseguir salvar vidas e o distanciamento de amigos e familiares devido a altas cargas de trabalho; o Brasil apresenta preponderância de sintomas depressivos em profissionais de enfermagem.

As condições atuais demonstram um fator significativo para o desencadeamento de problemas de saúde biológica, mental e social no enfermeiro; aumento do desgaste profissional devido à alta quantidade de pacientes, associada ao desconhecimento da doença e suas complicações; medo sentindo por parte do enfermeiro pela possibilidade de transmissão da doença a cônjuges, pais, filhos e outros parentes; escassez de recursos se mostra como importante fator para o adoecimento do enfermeiro, podendo gerar esgotamento profissional que se expressa através de mialgia, febre, depressão, rebaixamento do ânimo e queda imunológica.

Os fatores de risco associados ao adoecimento mental dos profissionais de saúde durante a pandemia englobam o risco de infecção durante a prática profissional, isolamento social, longas jornadas de trabalho, morte de pacientes, esgotamento físico e mental, sentimento de impotência em relação à cura de pacientes graves e falta de EPI's; sentimentos psicológicos englobam ansiedade, depressão, angustia, sentimento de desamparo, insônia, estresse e esgotamento; enfermeiras e técnicas do sexo feminino que trabalham na linha de frente foram identificadas como portadoras dos sintomas psicológicos mais graves.
SANTOS et al., $2020^{3}$
Avaliar, na literatura, a saúde mental dos COVID-19. 


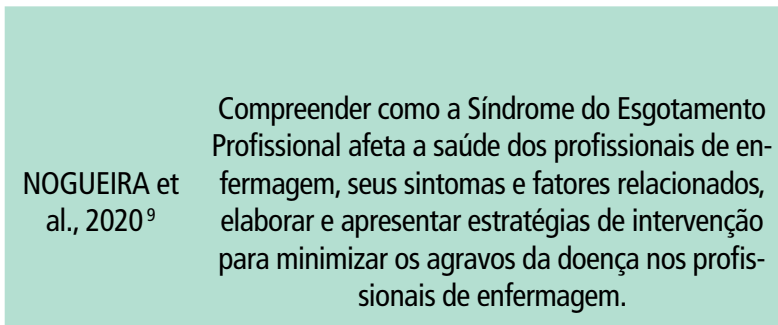

HUMEREZ et al., $2020^{4}$

Refletir sobre a saúde mental dos profissionais de enfermagem brasileiros no contexto da pandemia de COVID-19.
Uma das profissões mais afetadas pela síndrome do esgotamento profissional é a enfermagem; na área da saúde é considerada uma das profissões de maior risco para o desenvolvimento de doenças emocionais; os sintomas manifestados incluem falta de empatia, intolerância, indiferença, distúrbios do sono, fadiga crônica, irritabilidade, perda do

ânimo e manifestações depressivas; no atual cenária da pandemia houve um grande aumento na carga de trabalho e cuidados de prevenção, promovendo uma acentuação da exaustão física e emocional.
Os trabalhadores de enfermagem pressionados com atual situação apresentam elevados níveis de ansiedade, acrescidos do risco de adoecer, provocando severos problemas de saúde mental e aumentando os casos de síndrome de burnout, além de ansiedade, depressão e estresse associado; sentimentos mais declarados pelos profissionais foram ansiedade, devido à falta de EPI's, estresse, pelo alto nível de pacientes e mortes, medo de se infectar ou infectar algum familiar ou amigo, depressão e exaustão.

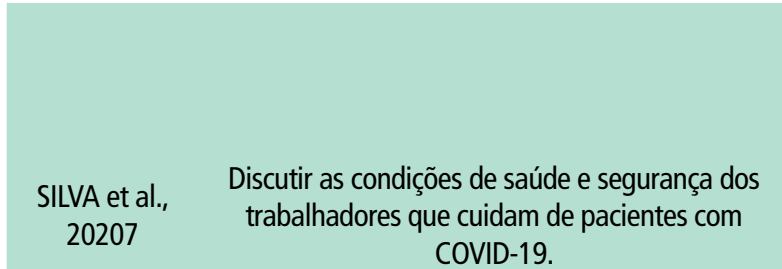
COVID-19.
A maioria dos profissionais de saúde que atendem um paciente contaminado ficam sujeitos a um alto risco de adquirir a doença; é clara a intensidade e a alta prevalência da infecção entre os trabalhadores da área da saúde, relatos de prevalência superior a $10 \%$ nessa população, chegando a $20 \%$ em italianos; o Brasil é ainda o pais em que mais morrem enfermeiros, 157 mortes de profissionais de enfermagem; aumento da carga de trabalho entre enfermeiros devido à escassez de médicos e outros profissionais da saúde.

Prevalência da ansiedade nos profissionais de enfermagem foi de $48,9 \%$ e a de depressão $25 \%$; maior parte da amostra composta de mulheres, pessoas acima de 40 anos, casadas ou em união estável; os profissionais de enfermagem com ansiedade são técnicos em cargo assistencial $(44,2)$, atuam em setores críticos $(55,8 \%)$, concursados $(44,2 \%)$ e traba-

Iham na área da saúde há mais de 10 anos (27,9\%).

Identificar a prevalência e fatores associados DAL'BOSCO et à ansiedade e depressão em profissionais de al., $2020^{13}$ COVID-19 em hospital universitário.

\footnotetext{
FONTE: Elaborado pelos autores
}

latos de prevalência superior a 10\% entre esses trabalhadores, chagando a $20 \%$ entre os italianos. O editorial ainda afirma que que cerca de 3.300 trabalhadores da saúde foram infectados na China e que 22, aproximadamente, haviam morrido até o final de fevereiro de 2020, dentre eles estava o profissional que alertou sobre o início da epidemia7.

Sobrecarga de trabalho, más condições de trabalho, falta de recursos, baixa valorização e remuneração são problemas enfrentados pela equipe de enfer- magem. Somado a isso, essa categoria profissional, no cenário atual, se encontrou na linha de frente da pandemia do COVID-19, esse fator se tornou um risco para o desenvolvimento de sintomas como ansiedade, depressão, medo, angustia e insônia2,1.

Cotidianamente a enfermagem é exposta a situações estressantes, como assistência a pacientes graves, cuidados intensivos e diretos, funções burocráticas e sobrecarga de trabalho, fatores que contribuem para o desen- volvimento da ansiedade e depressão, levando a um estado deletério da saúde mental13.

Diante da atual realidade, ocorreu uma elevação na carga de trabalho e nos cuidados de prevenção, acentuando a exaustão física e emocional. É importante frisar que esses profissionais tendem a se descuidar da sua saúde mental e passam por desafios como o enfrentamento do distanciamento social dos seus próprios familiares, medo, cansaço, angustia e a morte que passou a se tornar parte da 
rotina diária em meio ao caos instalado pela pandemia do COVID-199.

Durante momentos de pandemia é comum o surgimento do sentimento de horror e apreensão, além do aumento da preocupação com familiares e amigos. Os profissionais de saúde podem ter essa preocupação em níveis mais elevados devido a possibilidade aumentada de transmissão da doença infectocontagiosa para seus familiares6.

Vale ressaltar que os pacientes com diagnóstico de COVID-19 e seus familiares se encontram eu um momento de fragilidade emocional, e buscam nos profissionais de saúde um mecanismo de apoio em relação aos aspectos clínicos e emocionais, entretanto, percebe-se que os profissionais de saúde também são afetados pelo contexto no qual estão inseridos, podendo refletir na capacidade de atenção humanizada aos pacientes3.

A enfermagem executa tarefas exaustivas, além de jornadas de trabalho extensas e acompanhadas de plantões extenuantes, o que aumenta a suscetibilidade desses profissionais ao risco de desenvolvimento da SB. Diante da atual pandemia causada pelo COVID-19 os profissionais de enfermagem vêm sendo desencorajados a manterem relações próximas com outros indivíduos, conduzindo ao aumento do sentimento de isolamento e cansaço relacionado ao trabalho devido aos protocolos de utilização e remoção dos EPI's, o que dispõe de um tempo considerável dentro do ambiente laboral12.

A vivência dos enfermeiros na sistematização do cuidado ao paciente com COVID-19, acarreta consequências psicológicas negativas para o profissional tais como fadiga, desconforto e desamparo, desencadeados, principalmente, pela alta carga de trabalho e limitação dos EPI's em meio a pandemia. É importante ressaltar o medo que esses profissionais têm de adquirirem o vírus e colocarem seus familiares e amigos expostos, fator esse que culmina no estabelecimento do isolamento social, sendo deletério para à saúde mental3.

O elevado número de profissionais contaminados e afastados do ambiente laboral leva à uma sobrecarga de trabaIho ainda maior para as equipes de saúde, contribuindo de forma importante para o esgotamento psíquico da equipe de enfermagem. O estresse ocupacional é considerado um importante indicador de exaustão psíquica relacionado ao enfrentamento da pandemia e tem gerado algumas incertezas refletindo na saúde mental dos profissionais de enfermagem 2 .

\section{CONCLUSÃO}

É evidente que a enfermagem trabaIha em um cenário de cargas horárias exaustivas, más condições de trabalho, deficiência no dimensionamento de pessoal, poucos recursos materiais, desvalorização e baixa remuneração. Diante da pandemia causada pelo COVID-19 esse canário se tornou ainda pior, uma vez que a equipe de enfermagem é uma das categorias profissionais que estão na linha de frente prestando assistência à população.

Esses fatores, associados ao medo de transmitir a doença para familiares e amigos, o isolamento social e a morte como parte do cotidiano aumentaram ainda mais a sobrecarga física e psicológica sobre esses profissionais, aumentado o risco de desenvolvimento de problemas psicológicos e causando efeito deletério à saúde mental.

\section{Referências}

1. Ribeiro AP, Oliveira GL, Silva $L S$, Souza ER. Saúde e segurança de profissionais de saúde no atendimento a pacientes no contexto da pandemia de Covid-19: revisão de literatura. Rev Bras Saude Ocup 2020;45:e25. ISSN: 23176369 (online) http://dx.doi.org/10.1590/2317-6369000013920

2. Moreira AS, Lucca SR. Apoio psicossocial e saúde mental dos profissionais de enfermagem no combate à COVID-19. Enferm. Foco 2020; 11 (1) Especial: 155-161.

3. Santos WA, Beretta LL, Bruna SL, Silva MAP, Cordeiro GP, França EM. The impact of the COVID-19 pandemic on the mental health of healthcare workers: integrative review. Research, Society and Development, v. 9, n. 8, e190985470, 2020 (CC BY 4.0) | ISSN 2525-3409 | DOI: http://dx.doi.org/10.33448/rsdv9i8.5470

4. Humerez DC, OhI RIB, Silva MCN. Saúde mental dos profissionais de enfermagem do Brasil no contexto da pandemia do COVID-19: ação do Conselho Federal de Enfermagem. Cogitare enferm. 25: e74115, 2020. dx.doi. org/10.5380/ce.v25i0.74115

5. Coronavírus Brasil [página da internet]. Painel Coronavírus [acesso em 12 de outubro de 2020]. Disponível em: https://covid.saude.gov.br/

6. Júnior BSS, Mendonça AEO, Araújo AC, Santos RC, Neto FAD, Silva RAR. Pandemia do Coronavírus: estratégias amenizadoras do estresse ocupacional em trabalhadores da saúde. Enferm. Foco 2020; 11 (1) Especial: 148-15.

7. Silva LS, Machado EL, Oliveira HN, Ribeiro AP. Working conditions and lack of information on the impact of COVID-19 among health workers. Rev Bras Saude
Ocup 2020;45:e24. ISSN: 2317-6369 (online) http://dx.doi.org/10.1590/23176369000014520

8. Silva MO, Ribeiro AS. Nurses on the front line of the combat to COVID-19: professional health and user assistance. Research, Society and Development, v. 9, n. 8, e172985241, 2020 (CC BY 4.0) | ISSN 2525-3409 | DOI: http://dx.doi. org/10.33448/rsd-v9i8.5241

9. Nogueira KA, Costa KGS, Monteiro ACM, Desiderio NLM, Ferreira L, Queiroz GF, Refrande SM, Santos JL. Síndrome do esgotamento profissional na enfermagem em tempos de COVID-19. A enfermagem centrada na investigação científica 6 [livro eletrônico]. Ponta Grossa, PR: Atena, 2020.

10. PRISMA. Transparent Reporting of Systematic Reviews and Meta-Analyses [página da internet]. Disponível em: http://www.prisma-statement.org/ 11. Downs SH BN. The feasibility of creating a checklist forthe assessment of the methodological quality both of randomi-sed and non-randomised studies of health care interventions. J Epidemiol Community Heal. 1998.

12. Pereira MD, Torres EC, Pereira MD, Antunes PFS, Costa CF. Emotional distress of Nurses in the hospital setting in the face of the COVID-19 pandemic. Research, Society and Development, v. 9, n. 8, e67985121, 2020 (CC BY 4.0) | ISSN 2525-3409 | DOI: http://dx.doi.org/10.33448/rsd-v9i8.5121

13. Dal'Bosco EB, Floriano LSM, Skupien SV, Arcaro G, Martins AR, Anselmo ACC. Mental health of nursing in coping with COVID-19 at a regional university hospital. Rev Bras Enferm. 2020;73(Suppl 2): e20200434 http://dx.doi. org/10.1590/0034-7167-2020-0434 